enrolled in a youth leadership program in rural Guatemala. Pre-/post-testing was conducted.

Results Pre-/post-testing revealed increased injury prevention knowledge (76\% to $96 \%$ ) and self-confidence to implement community injury prevention strategies (50\% to 90\%) among participants. Participant feedback from the course was positive: $60 \%$ endorsed that all topics were comprehensively covered; however $20 \%$ desired more education on drowning prevention and road traffic injury. Ninety percent of adolescents anticipated positive safety behavior change post-course.

Discussion and Learning Outcomes Our modified Adolescent Injury Prevention Program was well-accepted by a class of Guatemalan adolescent learners and was efficacious at increasing their injury prevention knowledge and self-confidence. This novel approach of actively engaging adolescents in injury prevention programs could lead to increased adolescent safety behaviors, while building capacity among this high-risk population and their communities.

\section{P4.007 BACK SEAT SAFETY BELT USE AND CHILD RESTRAINT SEAT USE IN JAPAN}

Mellisa Ogasawara*, Atsuko Chiba, Kie Kawauchi, Yoshihide Sorimachi. Aomori University of Health and Welfare, Aomori, Japan

\subsection{6/injuryprev-2021-safety. 210}

Background A national survey reported driver and front passenger seat belt use to be high in Japan, 98.9\% and 95. 9\% respectively in 2019. On the other hand, back seat safety belt use is alarmingly low at $39.2 \%$. Child restraint seat (CRS) use has been mandatory since 2000, yet the survey reported the rate of use in 2019 to be only $70.5 \%$ for children under the age of 6 . Additionally, the rate of use for 5 -year-old children is dangerously low at $48 \%$. The purpose of this study was to determine rear passenger seat belt use and CRS use on shortdistance trips on local roads in a regional area of Japan.

Methods A cross-sectional survey using self-report questionnaires was performed at 78 nursery schools throughout Aomori prefecture. A descriptive analysis was performed on selected variables.

Results A total of 3021 valid responses (71.1\% response rate) were returned. The number of respondents who always wore a seat belt traveling short-distance on local streets as a backseat passenger was significantly low (25.7\%). Consistent CRS use on short trips using local roads was also considerably low with an average of $72.7 \%$.

Conclusion Rear passenger seat belt use is very low, as is CRS use on short-distance trips on local roads in Aomori prefecture. Parents' seat belt use as a backseat passenger may be affecting their behavior toward CRS use.

Learning Outcomes Interventions to increase the protection of children as passengers may need to address parents' perceptions of rear-seat safety.

\section{P4.008 BEYOND SUPERVISION: LINKING SIBLING RELATIONSHIP QUALITY AND SCHOOL-AGED CHILD INJURIES}

Caroline Piotrowski*, Julie-Anne McCarthy, Lynne Warda. University of Manitoba, Winnipeg, Canada

10.1136/injuryprev-2021-safety.211
Background While family size and sibling supervision have both been shown to influence child injury risk, the role of sibling relationship quality in child injury has not been investigated. Our goal was to investigate potential linkages between child injuries and the quality of sibling relationships.

Methods Seventy-nine families with two school-aged children aged seven and ten years on average were recruited from the community; $54 \%$ were female. Children reported on the quality of their sibling relationships and parents reported on the frequency of minor child injuries within the past three months, as well as their supervision attitudes.

Results Younger siblings in antagonistic relationships characterized by high hostility and low warmth incurred significantly more minor injuries; this was especially the case when age spacing between siblings was larger. Higher parental confidence in younger siblings was significantly related to fewer minor injuries for older, but not younger siblings.

Conclusions Sibling relationship quality played a significant role in injury risk for school-aged children, particularly when sibling age spacing was larger. Therefore, the quality of sibling relationships should be taken into account in future research, as well as in home injury prevention programs. Parental confidence in younger siblings was linked to fewer older sibling injuries, underlining the importance of understanding the interconnected nature of family dynamics on child injury.

Learning Outcomes Antagonistic sibling relationships were significantly associated with more minor injuries for younger siblings, especially when sibling age spacing was larger. Parental confidence in younger siblings was related to fewer older sibling minor injuries.

\section{P4.009 EPIDEMIOLOGY OF SCHOOL INJURIES AMONG MIDDLE SCHOOL STUDENTS IN KANDY, SRI LANKA}

Samidi Navaratna*, Samath Dharmaratne. Faculty of Medicine, University of Peradeniya, Sri Lanka, Kandy, Sri Lanka

\subsection{6/injuryprev-2021-safety.212}

Background School injuries are recognized as a preventable public health problem. No study has been conducted to find the incidence of school injuries in Sri Lankan schools up to date.

Methods A sample of 820 students were recruited using multistage cluster sampling from an education division in Kandy. A well-structured, pre-tested, interviewer-administered questionnaire was used to gather information on unintentional school injuries that took place in the preceding 1 month. Injury severity was assessed using the Abbreviated Injury Score (AIS). The statistical significance of the associations was tested using the chi-square test.

Results The event-based injury incidence rate was 25.37 per 100 students per month (95\% CI: 22.04-29.06). A majority $(31.8 \%)$ of the injury events occurred during a sports event. Some contributory factors identified were overexertion (32.2\%), starvation (19.7\%), and lack of maintenance of the premises (15.4\%). Common injury types were abrasions (33.5\%) and lacerations (14.7\%). Lower extremities $(50.7 \%)$ were affected most. The majority of injuries (94.7\%) fell to AIS 1, whilst $5.3 \%$ fell to AIS 2. Being a male $(\chi 2=22.6$, 
$\mathrm{p}<0.001)$, a member of $\geq 1$ school sports teams, $(\chi 2=11.6$, $\mathrm{p}=0.001)$, and involvement in a physical fight during the last 30 days $(\chi 2=5.7, p=0.01)$ were some of the risk factors for sustaining an injury. Only $42.3 \%$ had received first aid at school.

Conclusions \& Learning Outcomes Event-based school injury incidence is very high in the study area. Recommendations can be made to adopt safety measures during sports, proper maintenance of the school premises and improvement of first aid facilities.

\section{P4.010 SCOPING REVIEW OF ELOPEMENT BEHAVIOR AMONG CHILDREN WITH AUTISM}

Lise Olsen*, Crystal Shannon, Desiree Thompson, Catie Balehowski. University of British Columbia, Okanagan, Kelowna, Canada

\subsection{6/injuryprev-2021-safety.213}

Background Recreation for children with living with Autism Spectrum Disorder (ASD) is important for improving health and quality of life, however, children with ASD and their families experience many barriers to activity participation. Child elopement behavior is a key barrier that poses a safety concern for parents and is associated with injury occurrence.

Methods We conducted a scoping review, guided by Arksey and O'Malley's framework, to assess the literature on elopement among children with ASD. Search procedures were developed in consultation with university librarian and included searches of five data bases, grey literature, and handsearching. Included articles were those addressing elopement among children 0-19 years with ASD. Abstracts and full text articles were assessed by two reviewers and a third reviewer arbitrated disagreements.

Results A summary of search procedures and key study findings will be presented. Key findings pertain to: nature and patterns of elopement behavior and associated injury outcomes, factors associated with elopement behavior, and preventive intervention approaches and outcomes.

Conclusion This assessment of current literature promotes understanding of elopement behavior among children with ASD, related factors and preventive approaches. This research supports SDG \#10 for reduced inequalities through better understanding of elopement to inform strategies for promoting inclusive and safe recreation participation for children with ASD.

Learning Outcomes Participants will gain understanding of elopement as a safety issue and barrier to equitable recreation participation for children with ASD. Participants will gain knowledge of current research findings about elopement and its prevention.

\section{P4.011 COMMUNITY-LEVEL ESTIMATES OF DEATH DUE TO INJURIES IN MAKWANPUR DISTRICT, NEPAL}

\footnotetext{
${ }^{1}$ Santosh Bhatta*, ${ }^{1}$ Julie Mytton, ${ }^{2}$ Dhruba Adhikari, ${ }^{2}$ Sunil Raja Manandhar, ${ }^{3}$ Elisha Joshi, ${ }^{3}$ Sumiksha Bhatta, ${ }^{4}$ Sunil Kumar Joshi. 'University of the West of England, Bristol, UK; ${ }^{2}$ Mother and Infant Research Activities, Kathmandu, Nepal; ${ }^{3}$ Nepal Injury Research Centre, Kathmandu Medical College Public Limited, Kathmandu, Nepal; ${ }^{4}$ Department of Community Medicine, Kathmandu Medical College Public Limited, Kathmandu, Nepal
}

10.1136/injuryprev-2021-safety.214
Background Availability of injury related mortality data can play a vital role in planning health services for injury prevention and monitoring progress towards Sustainable Development Goal 3.4. In the absence of a robust death registration system, this study explored the feasibility of a model to identify injury deaths occurring outside hospital in rural Nepal.

Methods Data were collected prospectively between February 2019 and January 2020, in two rural municipalities in Makwanpur district. Female Community Health Volunteers notified all deaths in their area to the local Health-Post. Trained data collectors identified the injury-related deaths and invited relatives of the deceased to take part in a structured face-to-face interview.

Results Over one year, 67/451 (14.9\%) deaths were identified as secondary to an injury in the two study areas (injury mortality rate 104/100,000 population). The rate of community injury deaths was higher in males $(128 / 100,000)$ than females (80/ $100,000)$ with a median age at death of 40 years $(S D=20.8$, range 2 to 93). Community injury deaths were most commonly secondary to suicide $(65 / 100,000)$, followed by unintentional injury $(36 / 100,000)$ and assault/violence $(3 / 100,000)$.

Conclusion Large numbers of injury deaths occur outside hospital, and these appear more likely to be due to intentional than unintentional harm. These findings are a valuable additional source of information for engaging stakeholders, developing targeted injury prevention interventions and informing policies.

Learning Outcomes Community deaths secondary to injuries are common and will be missed in hospital-based data collection systems. The mechanism of injury may be different for community compared to hospital cases.

\section{P4.012 A BASIC SURVEY ASCERTAINING DETAILS RELATED TO FALLS ON STAIRS OR ESCALATORS}

Deborah Hilton*. Deborah Hilton Statistics Online [http://sites.google.com/site/ deborahhilton/], ASHWOOD, Australia

\subsection{6/injuryprev-2021-safety.215}

Background The Australian Institute of Health and Welfare state that falls account for $41 \%$ of all hospitalised injuries. In Melbourne City, at various train stations there are extremely long escalators and alarmingly one sign previously displayed states there are approximately 2-3 falls/week.

Methods A general convenience survey [ $n=125]$ was distributed to ascertain the percentage of people who've fallen down stairs and/or escalators. Additional questions related to rain, high risk footwear, poor lighting and how many falls they have had. Following on from that regardless of whether or not they'd had a fall, survey participants were asked about fear of falling and/or whether they felt uncomfortable around crowds on stairs or escalators.

Results 82 survey forms were returned. 29\% of people had fallen, with $16 \%$ of those people indicating rain, $16 \%$ in either high heels or thongs, while $25 \%$ indicated poor visibility. of those whom had fallen the estimate of the number of falls down stairs or escalators ranged from 1-20, with the average being 3 falls.

$39 \%$ of respondents irrespective of whether or not they had fallen had a fear of falling, while $43 \%$ felt uncomfortable around crowds. The age groups were 33\% [20-29 yrs], 8\% 